\title{
Modular and extensible lesson on fiber optics for youths
}

\section{Nicholas H. Wong, Amy S. K. Tong, Matthew Posner, Andrea Ravagli}

Nicholas H. L. Wong, Amy S. K. Tong, Matthew T. Posner, Andrea Ravagli, "Modular and extensible lesson on fiber optics for youths," Proc. SPIE 10452, 14th Conference on Education and Training in Optics and Photonics: ETOP 2017, 104520S (16 August 2017); doi: 10.1117/12.2266445

SDIE Event: 14th Conference on Education and Training in Optics and Photonics, ETOP 2017, 2017, Hangzhou, China 


\title{
Modular and extensible lesson on fiber optics for youths
}

\author{
Nicholas H. L. Wong, Amy S. K. Tong, Matthew T. Posner, and Andrea Ravagli \\ Optoelectronics Research Centre, University of Southampton, Southampton, SO17 1BJ, UK
}

\begin{abstract}
Fiber optics and its application in telecommunications are rarely encountered by students until they reach tertiary education. While some secondary/middle school curricula may include coverage of basic geometrical optics concepts such as reflection and refraction, few if any go further to elaborate on how these eventually relate to global telecommunications. One could say that the science is made accessible for early-stage students, but discussions about applications are often reserved till later stages. In working through a PhD student-led optics educational outreach program called the "Lightwave Roadshow", we have observed, via engagements with young students and the public at school visits and fairs, that many youths (as well as parents) do have a basic appreciation that the internet is somehow based on light signals. However, few know how the two are related, much less how they work. To address this, our team of 'ambassadors' in the Lightwave program has designed a self-contained lesson to introduce youths, aged 11 to 18 years, to fiber optics and optical fiber communications, drawing inspiration from various educational resources such as LASER Classroom ${ }^{\mathrm{TM}}$ and the Exploratorium ${ }^{\circledR}$. The lesson is modularized into several parts, starting with using light to communicate Morse code, and then going into advanced concepts, such as total internal reflection and multiuser communications based on wavelength-division multiplexing. The latter can be treated as extensions whose inclusion can be tailored based on the youths' educational levels. A feature of this lesson is that it takes a more phenomenological than theoretical approach, and uses materials that are easily obtainable or craftable as well as interesting for youths, including colored gelatin, LED sources, and water as a waveguide. We outline a lesson and pedagogical method which contains hands-on experiments that can be carried out by educators in formal or informal classes, students learning independently, or optics and photonics student chapters and groups doing educational outreach. The experiments within were first demonstrated at the 2016 OSA Frontiers in Optics/Laser Science conference.
\end{abstract}

Keywords: Fiber optics, optical communications, optics education, curriculum development, pedagogical tools, educational outreach, student chapter, STEM

\section{INTRODUCTION}

Shortages of skilled labor in science, technology, engineering and mathematics (STEM) sectors have prompted much research and funding into STEM education. ${ }^{1}$ Studies have shown that life aspirations of youths are formed during the primary and secondary school years (before the age of 14 years), and have identified early intervention as a possible solution to the STEM issue. ${ }^{2}$ Hence, many university-based educational outreach programs, including our own "Lightwave Roadshow", ${ }^{3}$ target young students to engage them with science through classroom-based hands-on workshops, and aim to get them interested in STEM from an early age. ${ }^{4}$

Through our outreach interactions, we have found that youths respond more enthusiastically to teaching when the concepts being taught have direct relevance to their life experiences. In the optics context, an example of a pervasive application is optical fiber communication which forms the backbone of the ubiquitous internet. However, applied fiber optics receives little if any coverage in school curricula. The United Kingdom (UK) national curriculum does include a short section on refraction, ${ }^{5}$ though this is only taught at the Years 10-11 levels (ages 14-16 years). In order to permeate optical fibre communications into earlier levels, we have thus devised a lesson that is modularized into three parts. The first module, which covers encoding and introduces basic communications using flashlights, can be taught to younger ages as a self-complete primer. For older students, the subsequent modules can function either as extensions or even standalone topics. The modules emphasize experimental exploration over theory and have been designed to work with equipment that is easily obtainable, craftable, and hence replicable.

A brief background of our outreach and the lesson is provided in Sec. 2. Thereafter, we describe the modules in detail in Sec. 3. Finally, we review some past workshops and conclude the paper in Secs. 4 and 5, respectively.

Further author information: (Send correspondence to N.H.L.W.)

N.H.L.W.: E-mail: nhlw1g12@orc. soton . ac. uk, Telephone: +44 2380593924

14th Conference on Education and Training in Optics and Photonics: ETOP 2017, edited by Xu Liu,

Xi-Cheng Zhang, Proc. of SPIE Vol. 10452, 104520S · @ 2017 ICO, IEEE, OSA, SPIE

CCC code: $0277-786 X / 17 / \$ 18 \cdot$ doi: $10.1117 / 12.2266445$

Proc. of SPIE Vol. 10452 104520S-1 


\section{BACKGROUND}

\subsection{Outreach program}

The Lightwave Roadshow is an optics educational outreach program, part of the University of Southampton's Optics and Photonics Society (OPSoc). OPSoc is run by and for (mostly $\mathrm{PhD}$ ) students of the university and comprises student chapters of The Optical Society (OSA), SPIE, and the Institute of Electrical and Electronics Engineers (IEEE) Photonics Society. ${ }^{6}$ The Roadshow, established in $1998,{ }^{3}$ engages with youths to teach them about optics via a pedagogy based on interaction with experiments. These are conducted or supervised by graduate student demonstrators or 'Lightwave Ambassadors'. Over time, the Roadshow has expanded its reach to youths in schools all over the Hampshire, England region. We have also gone beyond the traditional traveling classroom workshop style to other engagement formats, including STEM fairs, ${ }^{4}$ on-campus workshops for visiting schools, ${ }^{7}$ national flower shows, ${ }^{8}$ international academic conference exhibitions, ${ }^{9}$ and most recently, cathedrals. ${ }^{10}$ Apart from youths, these have additionally enabled us to engage with parents, educators, academics, industry professionals, other student chapters, and the general public.

\subsection{Lesson scope}

The lesson detailed in this paper focuses on the theme of fiber optic communications and has been formulated to be modular and extensible so that it can be appreciated by students of diverse ages from 11 to 18 years (Key Stages 3 to 5 of the UK national curriculum ${ }^{11}$ ). Throughout the modules, hands-on experiments and demonstrations are included to arouse the interest of students in the field of optics. These use equipment sourced from off-the-shelf components and can be easily replicated by the teachers and students. This lesson can also be modified for use at fairs and exhibitions.

Three modules covering various topics are presented, with subsequent modules building upon earlier ones and targeting advanced ages. Module 1 begins with an introduction to communications and representing information as light signals. This leads to module 2 which concerns the concepts of refraction and refractive index, where students learn about Snell's law and total internal reflection as the main guidance mechanism in optical fibers. For advanced students, multiuser communications is taught in module 3, where the concept of multiplexing many user channels into the same shared fiber medium is introduced. The wavelength-division multiplexing technique and receiver-side channel filtering is discussed. The next section details these modules.

\section{MODULES}

This section outlines the contents of the various modules taught. Each module includes some basic theory (as would be delivered to pupils) followed by one or more hands-on activities. Target ages are listed in parentheses.

\subsection{Module 1: information (for ages 11+)}

\subsubsection{Coding and modulation}

Information is represented as a sequence of bits and in basic binary systems, there are two possible states for each bit. For instance, computers use digital 1 and $\theta$ to represent logical TRUE and FALSE, respectively. Two things are required to communicate information. Firstly, there needs to be a system to assign meaning to bit sequences. This is known as encoding. A well-known system that many youths may have heard of is Morse code. Here, the two binary states are represented as dots $\bullet$ and dashes -, and each letter and numeral in the alphanumeric set (i.e. A to Z and 0 to 9) is encoded as a distinct sequence of these. Tab. 1 lists the internationally used Morse codes. As an example, the universal distress signal "SOS" is encoded as $\bullet \bullet---\bullet \bullet$.

Table 1: International Morse code chart.

\begin{tabular}{|c|c|c|c|c|c|c|c|c|c|c|c|c|c|c|c|}
\hline A & $\bullet-$ & $\mathrm{F}$ & $\bullet \bullet-\bullet$ & K & $-\bullet-$ & $\mathrm{P}$ & $\bullet--\bullet$ & U & $\bullet \bullet-$ & Z & $--\bullet$ & 1 & $\cdot----$ & 6 & $\bullet \bullet \bullet \bullet$ \\
\hline B & $-\bullet \bullet$ & G & $--\bullet$ & $\mathrm{L}$ & $\bullet-\bullet \bullet$ & Q & $--\cdot-$ & V & $\bullet \bullet \bullet-$ & & & 2 & $\bullet \cdot---$ & 7 & $--\bullet \bullet \bullet$ \\
\hline C & $-\bullet-\bullet$ & $\mathrm{H}$ & $\bullet \bullet$ & M & -- & $\mathrm{R}$ & $\bullet-\bullet$ & W & $\cdot--$ & & & 3 & $\bullet \bullet \bullet--$ & 8 & $---\bullet \bullet$ \\
\hline $\mathrm{D}$ & $-\bullet \bullet$ & I & $\bullet$ & $\mathrm{N}$ & $-\bullet$ & S & $\bullet \bullet$ & $X$ & $-\bullet \bullet-$ & & & 4 & $\bullet \bullet \bullet \bullet-$ & 9 & $----\bullet$ \\
\hline E & • & J & $\cdot---$ & $\mathrm{O}$ & --- & $\mathrm{T}$ & - & $\mathrm{Y}$ & $-\cdot--$ & & & 5 & ••••• & 0 & -- \\
\hline
\end{tabular}

Secondly, a physical means of conveying the two binary states is needed. This can be conveniently actualized, in the optical context, by turning a flashlight on or off, or in the Morse code example, by turning a flashlight on briefly (e.g. 
1 second) for a dot and turning it on for longer (e.g. 3 seconds) for a dash. ${ }^{12}$ In this way, a person sending a message by means of Morse code using a flashlight would be transmitting a sequence of long and short flashes. This is known as modulation. That is, the (encoded) message modulates the communications signal (e.g. light flashes) which then travels through a channel (e.g. air) to be perceived by a receiver (e.g. another person seeing the flashes).

\subsubsection{Class activity: Morse code}

In this activity, students work in pairs to send Morse coded messages to one another. Each pair is provided with a flashlight, a cardboard tube, a Morse code chart such as Tab. 1, a Morse code decoding tree chart as shown in Fig. 1, two pens or pencils, and several blank sheets of paper.

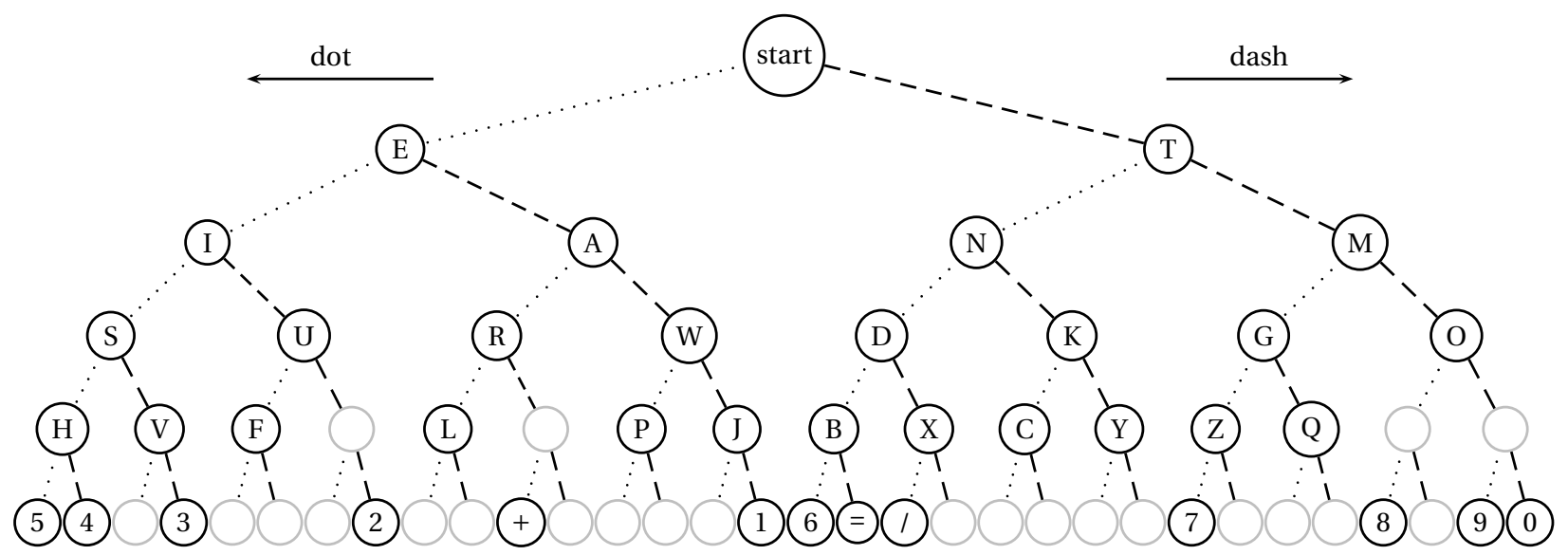

Figure 1: Morse code decoding tree.

In each pair, one student is assigned as the sender and the other, the receiver. The sender decides on a message unbeknownst to the receiver. S/he encodes the message in Morse code referring to Tab. 1 and may choose to discreetly write down the encoded sequence. The sender then transmits the encoded message by turning the flashlight on and off accordingly and shines the beam through the cardboard tube, which acts as a fiber channel, as depicted in Fig. 2a. The receiver looks at and records the sequence of flashes from the other end of the tube (i.e. the output). S/he then uses the chart in Fig. 1 to decode the message and verify it with the sender. For each encoded sequence, the receiver begins at 'start' and moves down the tree. Each dot or dash corresponds to a left or right traversal, respectively, to the next lower level. The node on which the traversal ends is the decoded character. For example, a 'W', which is encoded as $\bullet--$, would have a tree traversal beginning at 'start' and moving left $\rightarrow$ right $\rightarrow$ right. Although it is possible to use Tab. 1 for the decoding process, the reverse lookup is unordered and therefore cumbersome. Hence, the chart in Fig. 1 is recommended as an easier alternative. After one round, the sender and receiver can swap roles if time permits.

(a)

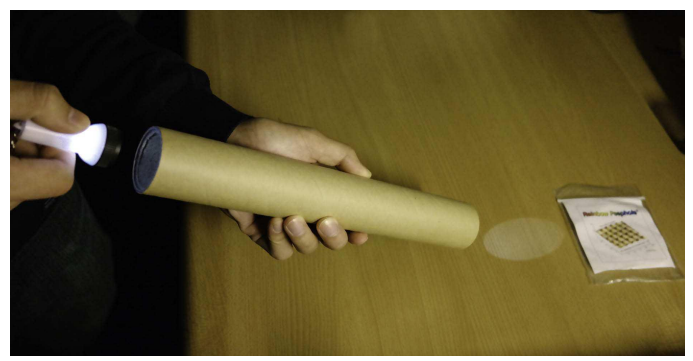

(b)

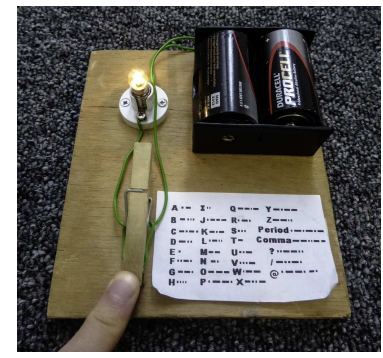

(c) Clothes peg

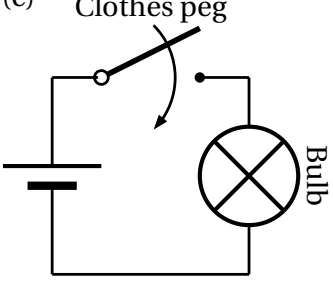

Figure 2: (a) Morse code class activity using a flashlight and cardboard tube. (b-c) Morse code circuit and schematic.

A number of tips can be considered. Firstly, the rule of no verbal communication should be articulated to the students. This ties the activity closer to reality, where verbal communication is not possible over long distances. Secondly, situations when the receiver decodes an incorrect message can be used as opportunities to introduce students to the existence of errors and the associated challenges of communications systems. For instance, a receiver mistaking a dot 
for a dash is analogous to a bit error in digital systems. Thirdly, students may discover that the manual process of sending dots and dashes is tedious and slow. This is an opportunity to highlight the need for ever faster high-speed transceiver systems, especially with exponentially increasing internet traffic. ${ }^{13}$ Lastly, it is helpful to show students and let them interact with a simple home-built Morse code circuit, such as in Figs. 2b-c, for them to appreciate how Morse code was telegraphically transmitted historically.

\subsection{Module 2: light guidance (for ages 14+)}

\subsubsection{Reflection and refraction}

Most students would already be familiar with the concept of reflection. Examples, such as bathroom mirrors, are given to reinforce the students' realization that it is a common perceptible phenomenon. The less conspicuous concept of refraction is also introduced. An example of a real-world occurrence, such as the perceived bending of a straight straw in a glass of water as in Fig. 3a, can be given.

(a)

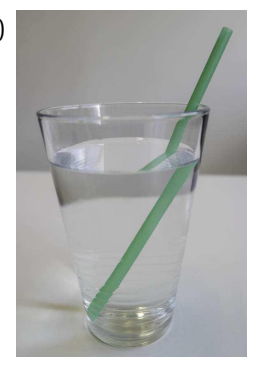

(b)

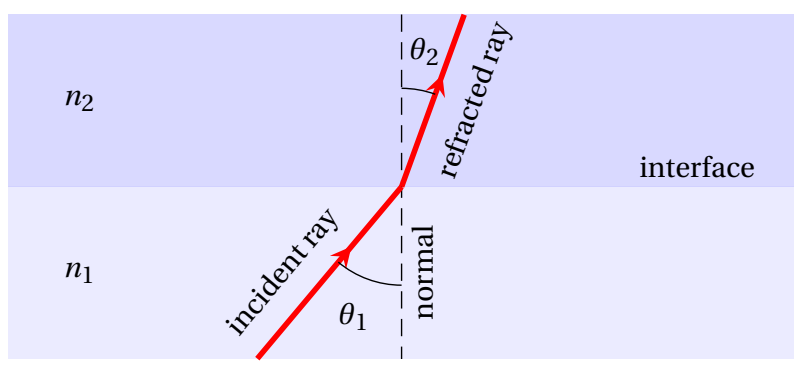

Figure 3: (a) Refracted straw image. (b) Refraction of light traveling through two materials of different refractive indices $n_{1}<n_{2}$.

Refraction is explained as a change in the speed of light as it travels between materials of different refractive indices. For young students, it is unnecessary (and inadvisable) to elaborate on the microscopic particle interactions that cause the change of speed. Rather, it suffices to mention that light slows down when travelling through a more optically dense material, which has a higher refractive index. The difference in refractive indices, and the resulting difference in light propagation speed, between two adjoining materials through which light travels causes it to bend at the interface, thus leading to refraction, as shown in Fig. 3b. The refractive indices $n$ of each material are related to the trajectory angles $\theta$ of light passing through them (measured from the normal to the interface) through Snell's law of refraction: ${ }^{12}$

$$
n_{1} \sin \theta_{1}=n_{2} \sin \theta_{2}
$$

The scenario in Fig. $3 \mathrm{~b}$ is typical of light passing from a material of lower refractive index, e.g. air, to one of higher refractive index, e.g. water. That is, $n_{1}<n_{2}$.

\subsubsection{Total internal reflection}

The case converse of Fig. 3b, i.e. when light travels from a higher to a lower refractive index, is also possible (see Fig. 4a). This time, the incidence angle $\theta_{1}$ is smaller than refraction angle $\theta_{2}$, since light travels faster after it reaches the material of lower index $n_{2}$. As the incidence angle is increased, so too will the refraction angle until is perpendicular to the

(a)

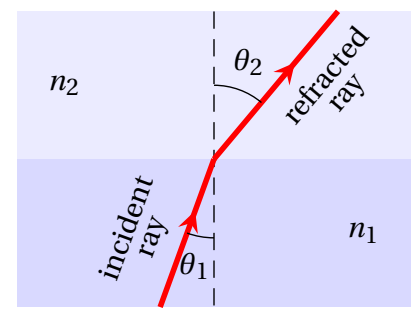

(b)

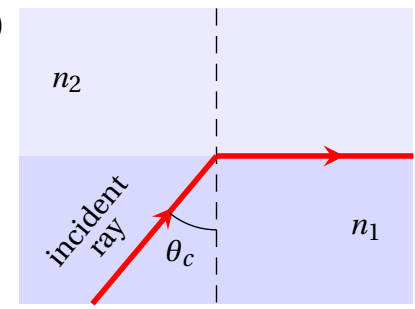

(c)

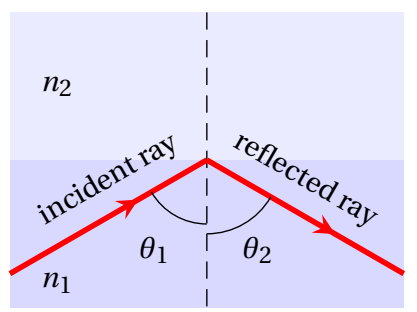

Figure 4: Light at a refractive index interface with $n_{1}>n_{2}$ : (a) refraction, (b) critical refraction, and (c) total internal reflection. 
normal, as in Fig. 4b. At this point, the incidence angle is known as the critical angle $\theta_{c}$. If the incidence angle is increased further than the critical angle, i.e. when $\theta_{1}>\theta_{c}$ as in Fig. $4 \mathrm{c}$, then no light is refracted and all light is instead internally reflected. ${ }^{14}$ The interface acts as if it were a mirror and the reflection angle $\theta_{2}$ is equal to $\theta_{1}$. This phenomenon is known as total internal reflection (TIR). ${ }^{15}$ It is emphasized that TIR can only occur for light traveling from within a material of higher refractive index to one of lower refractive index.

\section{Application: light guidance in an optical fiber}

If light travels in a material that is sandwiched between materials of lower refractive index, then it is possible that for certain ray angles, light will undergo TIR at all interface sides. In this way, it can be confined to the higher index material. ${ }^{14}$

This is the principle by which optical fibers work. A central circular glass core is surrounded by a glass cladding of slightly lower refractive index, as shown in Fig. 5. At appropriate angles, a light ray traveling within the core can continuously undergo TIR at all sides and thereby propagate from one end of the fiber to the other.

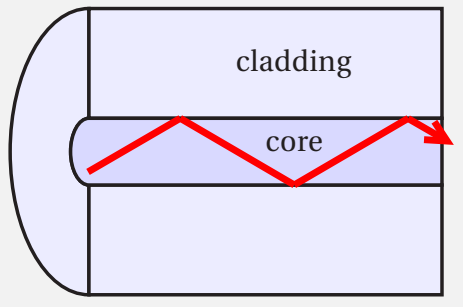

Figure 5: TIR in an optical fiber (crosssection).

\subsubsection{Class activity: measurement of refractive index}

The objective of this experiment is for students to measure the refractive index of gelatin. Students can work in small groups and each group is provided with a transparent square container prepared with clear gelatin, a $360^{\circ}$ protractor,* a slitted light-emitting diode (LED) source, ${ }^{\dagger}$ a scientific calculator, some pens or pencils, and an associated worksheet.

The experiment is set up as light passing between air and gelatin. From Eq. (1), the unknown refractive index of gelatin $n_{\text {gel }}$ can be determined from the known refractive index of air $\left(n_{\text {air }} \approx 1\right)$ as well as the angles of incidence and refraction which are to be measured. This is done by placing the gelatin container on the protractor, with the edge of the container on the $0^{\circ}$ to $180^{\circ}$ line of the protractor. A narrow beam from the LED source is shined at an angle through the edge of the container, at the point through the center of the protractor, as depicted in Fig. 6a. The higher $n_{\text {gel }}$ compared to $n_{\text {air }}$ causes the light to slow down and refract when traveling through the gelatin. By measuring the incidence and refraction angles, $\theta_{\text {air }}$ and $\theta_{\text {gel }}$, respectively, and rearranging Eq. (1), $n_{\text {gel }}$ can be calculated via

$$
n_{\mathrm{gel}}=\frac{\sin \theta_{\mathrm{air}}}{\sin \theta_{\mathrm{gel}}}
$$

This refractive index is typically about 1.34 to 1.40 . Depending on whether the students are familiar with trigonometry, it may be necessary to assist them in calculating the sines of angles using the scientific calculator.

(a)

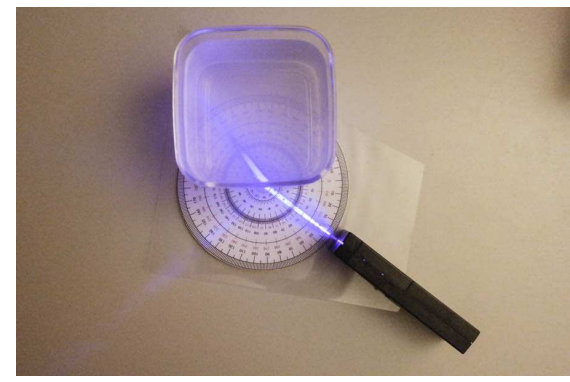

(b)

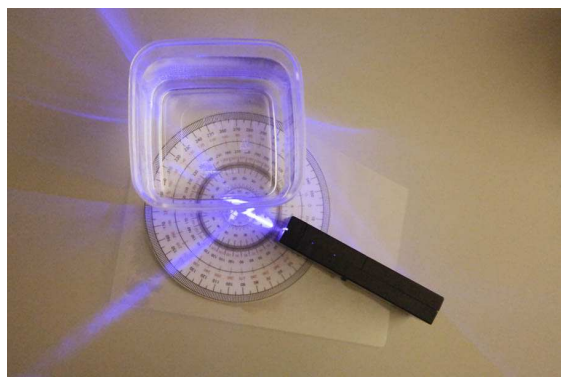

Figure 6: Refractive index measurements using (a) clear gelatin and (b) water.

A simple extension to the activity is for the students to measure the refractive index of water. The same aforementioned procedure is used, with the container holding water instead of gelatin, as shown in Fig. 6b. The refractive indices

\footnotetext{
${ }^{*}$ Such a protractor can be found in the Explore Optics OSA 100 Kit by LASER Classroom ${ }^{\mathrm{TM}} .{ }^{16}$

${ }^{\dagger}$ We used the Light Blox from the Light Blox Kit by LASER Classroom ${ }^{\mathrm{TM}} .{ }^{17}$
} 
of water $(\sim 1.33)$ and gelatin can then be compared and a teacher/demonstrator-led discussion can follow about how gelatin, being a solid, has an index closer to that of the glass in optical fibers. Although any color of light can be used for the measurements, it is important that the same color is used for both the gelatin and water cases, since different colors (wavelengths) of light travel at different non-vacuum speeds ${ }^{12,15}$ and will refract at slightly different angles.

\subsubsection{Class demonstration: light guidance using TIR}

Continuing from the refraction topic, the effect of TIR as discussed in Sec. 3.2.2 can be demonstrated. This can be achieved either by the students themselves by manipulating the incidence angle on the gelatin set-up as in Fig. 7a, or through a class demonstration of the Tyndall/Colladon experiment, ${ }^{6}$ where demonstrators guide laser light through a flowing water stream as in Fig. 7b. The latter requires a large transparent tank with a faucet, filled with water, a laser pointer, and a water collection bucket. A combination of both demonstrations is encouraged.

(a)

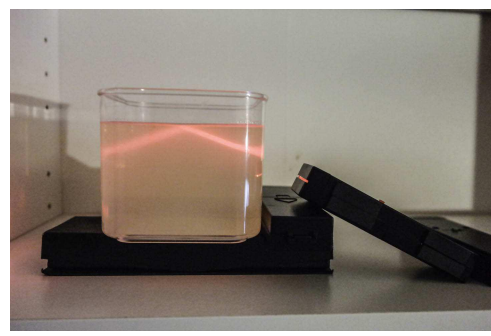

(b)

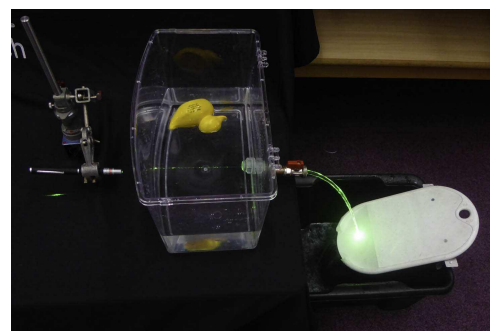

(c)

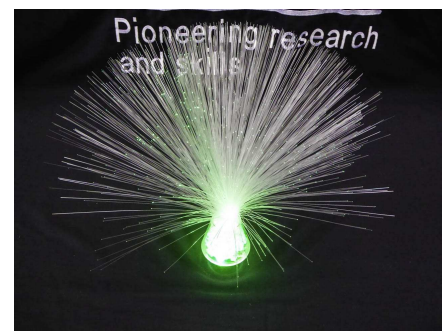

Figure 7: TIR demonstrations using (a) a container of clear gelatin and (b) a home-built Tyndall/Colladon experiment set-up. (c) A fiber fountain used as a teaching aid for TIR.

An additional useful tool is a commercial fiber fountain, like that shown in Fig. 7c. The hairlike plastic strands provide a safe emulation of real glass fibers. Moreover, its aesthetic nature can be utilized to gain student attention.

\subsection{Module 3: multiplexing (for ages 16+)}

The preceding modules covered communications and the related physics behind it in cases concerning only one sender and one receiver. A useful question to pose to students is, "how can multiple people, each communicating their own information, use the limited fiber resources at the same time?" The answer is multiplexing. Multiplexing is the transmission of multiple streams of information through a single medium by dividing it into multiple channels. ${ }^{12,18}$ In particular, this module covers wavelength-division multiplexing (WDM), where different channels are represented as different optical wavelengths (colors) and travel simultaneously through the same fiber medium.

\subsubsection{Wavelength-division multiplexing}

Fig. 8 portrays a simplified model of the WDM picture. Each sender, who may be communicating different types of data as illustrated by the various icons, ${ }^{\ddagger}$ uses a separate wavelength as indicated by the colored lines. These are all multiplexed (MUX) and transmitted simultaneously through the same fiber link. At the receiver side, the signals are demultiplexed (DEMUX) to separate each channel, and subsequently routed to each respective recipient.

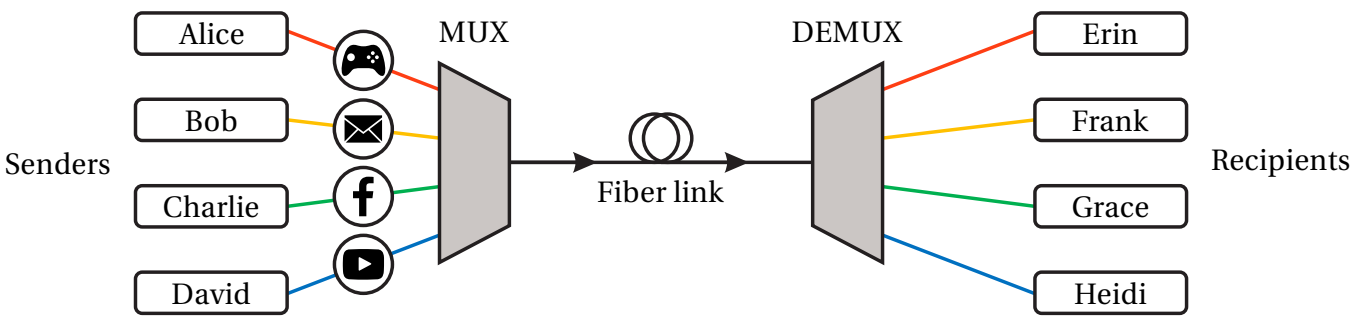

Figure 8: Multiuser system utilizing WDM on a single shared fiber link.

\footnotetext{
${ }^{\ddagger}$ The specific choice of icons here was made to relate to youths, as well as to highlight that certain types of information, e.g. data from gaming, also travels through the global fiber network.
} 


\subsubsection{Class activity: wavelength-division demultiplexing}

Demultiplexing is the process where the individual channels that were multiplexed together are separated at the receiver. ${ }^{14}$ Students learn about the separation of colors through filtering. They can be divided into small groups, with each group having a transparent container prepared with clear gelatin as per Sec. 3.2.3; three other transparent containers, each prepared with a different colored jelly; ${ }^{\S}$ and three slitted LED light sources emitting red, green, or blue.

In this activity, which was inspired by the "Laser Jello" experiment from the Exploratorium ${ }^{\circledR 19}$ and the "Transmission with Gummy Bears" activity from LASER Classroom ${ }^{\mathrm{TM}},{ }^{20}$ students first shine all three LED colors into the clear gelatin, noting that they are all transmitted, as shown in Fig. 9a. Thereafter, they experiment with different colored jellies to observe that a particular colored jelly transmits its correspondingly colored LED beam and not others, thus acting as a wavelength (channel) filter. Figs. $9 \mathrm{~b}-\mathrm{d}$ depict this effect, where switching among the colored jellies resembles the action of a tunable optical filter. ${ }^{18}$ It should be noted that because the beam colors are not exactly at the same wavelengths as the colors of the corresponding jellies, all beams are neither completely transmitted nor completely filtered out; rather it is the relative degree of transmission and filtering that matters for this pedagogy. For instance, the red jelly in Fig. 9b mostly transmits the red beam and mostly filters out the green and blue beams. In Fig. 9c, the purple colored jelly is actually closer in color to the red beam than the blue one and therefore transmits red more. Lastly, to put together the scenario of having both a multiplexed signal in a fiber and a receiver demultiplexer, the beams can be first shone through the clear gelatin (representing the fiber) and then through any of the colored jellies (representing the demultiplexer), as portrayed in Fig. 9e." This experiment can also be carried out using laser pointers instead of LED sources, in which case it is recommended to be demonstrated by experienced teachers/demonstrators, rather than by students working on their own.
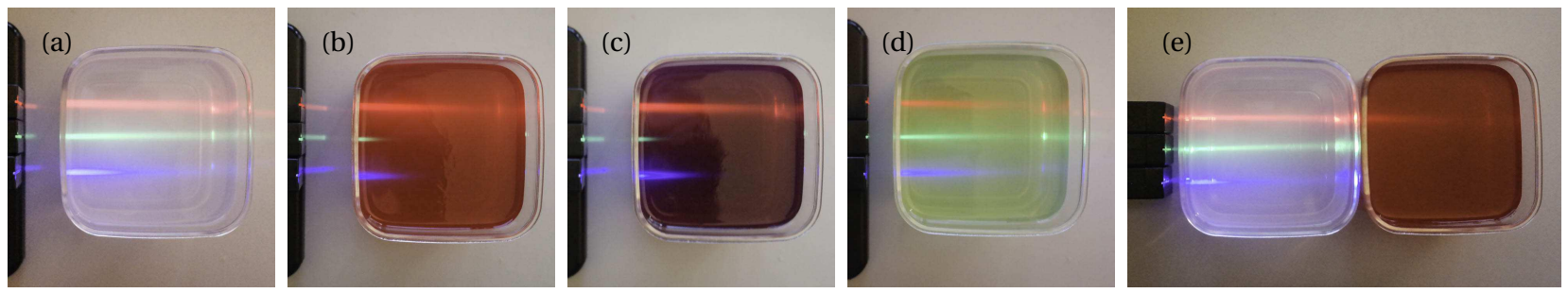

Figure 9: Multiple wavelength transmission through (a) clear gelatin and selective filtering using (b) red, (c) purple, and (d) green jellies. (e) Clear gelatin and colored jelly used to simulate a fiber and demultiplexer set-up.

\section{REVIEW OF PAST CLASSES AND DEMONSTRATIONS}

Modules in this lesson have been used in a few fairs and classes in the UK and abroad, and have received positive feedback. This section describes some of these experiences.

\subsection{Conference tabletop demonstration (modules 2 and 3)}

Prior to the modular formulation of this lesson, we first showcased the experimental elements of modules 2 and 3 at the 2016 OSA Frontiers in Optics/Laser Science conference. These, shown in Fig. 10a, were demonstrated on a $4 \mathrm{ft} \times 2 \mathrm{ft}$ tabletop and included the gelatin set-up for measuring refractive index, the Tyndall/Colladon experiment for showing TIR, and the colored jellies set-up for WDM. Attendees from academia and industry visited our table, and a common comment was of their intrigue with the diversity of optical effects that could be demonstrated using simple (mostly household) components. This positive reception prompted the creation of the modular lesson.

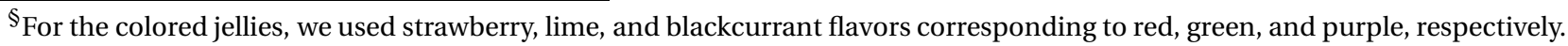
These choices were influenced by the use of correspondingly colored light sources, as well as store availability.

"We acknowledge that this set-up does not strictly model an actual multiplexed system, since the colored beams are spatially separated. Nevertheless, it suffices as an analogy to real systems.
} 
(a)

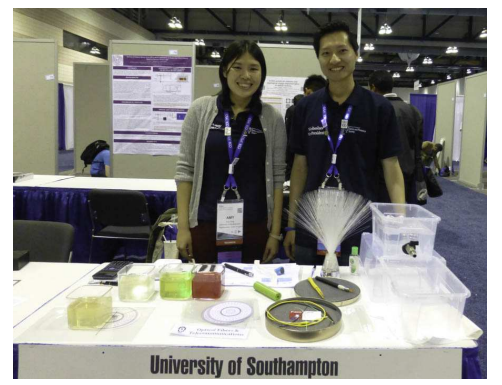

(b)

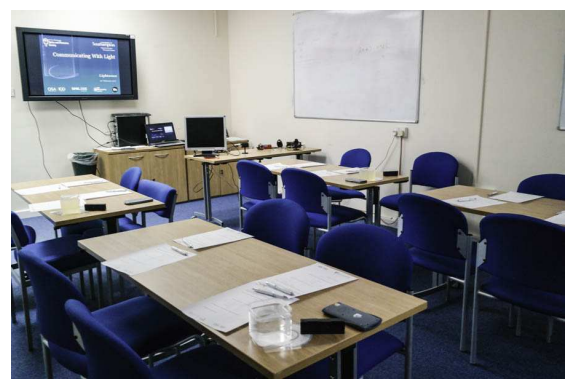

Figure 10: (a) Tabletop demonstration at the $2016 \mathrm{FiO} / \mathrm{LS}$ conference. (b) Classroom activity layout for a refractive index workshop.

\subsection{Class workshop on Morse code (module 1$)$}

Through our university's Widening Participation Programme,${ }^{6}$ we periodically organize optics workshops on our university campus for visiting schools. One example is our ( one-hour-long) "Communicating with Light" workshops, ${ }^{7}$ which teach about optical fiber communications at a basic level and incorporates elements from the modules in Sec. 3.

One of these workshops was run for Year 8 (12 to 13-years-old) students to educate them about optical communications. It was run three consecutive times on the same day, each for a different visiting school, for a total of 65 pupils. The first teaching objective was to enable the students to learn about how information can be encoded using light signals and to introduce Morse code. Following a short theoretical introduction as in Sec. 3.1.1, students then participated in the activity described in Sec. 3.1.2, where pairs practised Morse encoding messages with flashlights, using cardboard tubes as 'fiber' channels, and decoding the received messages. Worksheets were provided detailing instructions and suggested messages. Afterwards, the pros and cons of this communication method were discussed. The second objective, as an extension to modulation and coding, was to have students recognize the shortcomings of free-space optical communications and hence the motivation behind optical fibers. A set-up that included a live feed from a video camera being sent, over a free-space laser link, to a monitor ${ }^{6}$ was shown. By demonstrating that signals could be interrupted by using one's hand to block the free-space link, as well as explaining the need for guiding light around the curved surface of the earth, students appreciated the rationale for fibers. The third objective was to teach students about how optical fibers work. Owing to the young age range, this was done on a superficial level, where TIR was explained in simplistic terms of bouncing rays, without introducing refractive index. The Tyndall/Colladon experiment in Fig. 7b was demonstrated to show TIR in water, as an analogy to glass fibers.

A short multiple-choice question quiz was conducted and the students exhibited a good understand of the class, with $92 \%$ scoring $80 \%$ or higher. Some fiber research at the university was described to conclude the workshop, which was rated 3.5 on average by the students, using a 1 (very poor) to 5 (very good) scale.

\subsection{Class workshop on refraction and TIR (module 2)}

A separate "Communicating with Light" workshop was held for eight Years 7 to 11 (11 to 16-years-old) students, focusing on three teaching objectives. The first was to introduce pupils to optical communications. This was done with the same free-space video transmission set-up as in Sec. 4.2 and Ref. 6. Fiber optics was subsequently presented as a solution to overcome the limitations of free-space communications. The second objective aimed to teach refraction and refractive index. A brief theoretical overview as per Sec. 3.2.1 was given. To solidify understanding, the activity in Sec. 3.2.3 was carried out, requiring pupils, working in groups of 2-3, to determine the refractive index of given samples of jelly and water (see Fig. 10b for classroom layout). Worksheets containing step-by-step instructions were provided, and demonstrators gave assistance as needed. The third objective was for students to learn about optical fiber guidance through TIR. The theory in Sec. 3.2.2 was discussed and the demonstrations in Figs. 7b-c were used to show TIR in water and plastic, respectively.

The class was enjoyed by the students, with an $8 / 10$ average rating ( $10=$ 'excellent'). The application of light in the internet and the theory behind it was well received. Interest was also shown for the hands-on activity. However due to the wide student age range, the younger pupils (aged 11-13 years) required more aid to complete the measurements. To finish the class, each demonstrator gave a three-minute presentation on their PhD research. From the feedback slips, it was seen that eight out of ten pupils were able to correctly name one research topic related to light. In addition, all the pupils raised the possibility to consider future study in photonics and to advise others likewise. 


\section{CONCLUSIONS}

We have outlined a lesson for youths on fiber optic communications, with age-appropriate modules that can be taught separately or together. We reviewed past workshops utilizing these modules and the participant feedback obtained will help for future improvements. It is hoped that this lesson may be helpful in the work undertaken by other educators.

\section{ACKNOWLEDGMENTS}

We wish to thank everyone in the Lightwave team. We acknowledge support from the Engineering and Physical Sciences Research Council grant EP/N00762X/1 (National Hub in High Value Photonic Manufacturing) and the University of Southampton's Faculty of Physical Sciences and Engineering for sponsoring researchers taking part in these events. We also thank the OSA, SPIE, and IEEE Photonics Society for funding outreach kits.

\section{REFERENCES}

[1] Morgan, R., Kirby, C., and Stamenkovic, A., “The UK STEM Education Landscape.” Royal Academy of Engineering, May 2016, www. raeng . org. uk/stemlandscape. (Accessed: 3 May 2017).

[2] Tytler, R., Osborne, J., Williams, G., Tytler, K., and Cripps Clark, J., [Opening up pathways: Engagement in STEM across the Primary-Secondary school transition], Australian Department of Education, Employment and Workplace Relations, Canberra, Australia (2008).

[3] Bricchi, E., Baggett, J. C., Guilhot, D. A., and Musgrave, I. O., “The lightwave road show,” Proc. SPIE 9663, 96631L (2003).

[4] Foreman, H. D., Parmigiani, F. R., Roelens, M. A., and Simpson, R. E., “The lightwave roadshow," Proc. SPIE 9664, $96641 \mathrm{U}(2005)$.

[5] "National curriculum in England: science programmes of study." GOV.UK, September 2013, www.gov.uk/gov ernment/publications/national - curriculum - in-england-science-programmes - of - study. (Accessed: 3 May 2017).

[6] Wong, N. H. L., Posner, M. T., and John, P. V., "The Lightwave program and roadshow: An overview and update," Proc. SPIE 9793, 97932V (2015).

[7] Posner, M. T., John, P. V., Wong, N. H. L., Mittal, V., and Nunez-Velazquez, M. M., "From school classes to UNESCO: IYL-enabled environments for tackling the STEM skills shortage through student-led outreach," Proc. SPIE 9946, 994607 (2016).

[8] Posner, M. T., John, P. V., Standen, D., Wheeler, N. V., van Putten, L. D., Soper, N., Parsonage, T. L., Wong, N. H. L., and Brambilla, G., "Reflecting Photonics: Reaching new audiences through new partnerships - IYL 2015 and the Royal Horticultural Society Flower Show," Proc. SPIE 9946, 994603 (2016).

[9] Wong, N. H. L., Posner, M. T., Mittal, V., Gray, D. R., and John, P. V., "Taking local optics outreach abroad for IYL 2015: administrative and logistical challenges and strategies," Proc. SPIE 9946, 99460F (2016).

[10] Posner, M. T., Jantzen, A., van Putten, L. D., Ravagli, A., Donko, A. L., Soper, N., Wong, N. H. L., and John, P. V., "Cathedral Outreach: Student-led Workshops for School Curriculum Enhancement in Non-Traditional Environments," Proc. SPIE, ETOP (2017). [Accepted].

[11] “The national curriculum." GOV.UK, April 2017, www. gov . uk/national - curriculum. (Accessed: 26 April 2017).

[12] Dobson, K., Grace, D., and Lovett, D., [Physics], HarperCollins Publishers, London, UK, 2nd ed. (2002).

[13] Cisco, "Cisco Visual Networking Index: Forecast and Methodology, 2015-2020." White Paper (2016).

[14] Saleh, B. E. A. and Teich, M. C., [Fundamentals of Photonics], Wiley, Hoboken, NJ, 2nd ed. (2007).

[15] Tur, M., "Optical fibers - basics," in [Advanced Fiber Optics], Thévenaz, L., ed., 1-27, EPFL Press, Lausanne, Switzerland (2011).

[16] “Explore Optics OSA 100 Kit." LASER Classroom, http://store . laserclass room. com/explore - optics - osa100 - kit/. (Accessed: 4 May 2017).

[17] “Light Blox Kit: International Year of Light Edition.” LASER Classroom, http : //store. laserclass room . com/te ch-light- lab-light-science-kit. (Accessed: 4 May 2017).

[18] Agrawal, G., [Fiber-Optic Communication Systems], Wiley, New York, NY, 3rd ed. (2002).

[19] "Laser Jello." Exploratorium, www. exploratorium. edu/snacks/laser - jello. (Accessed: 3 May 2017).

[20] “Explore Optics: OSA Curriculum Guide.” LASER Classroom, www. laserclass room. com/wp - content/uploads / 2017/04/0SA_2017ActivityGuide.pdf. (Accessed: 4 May 2017). 